\title{
HERMENÉUTICA Y CRÍTICA
}

\author{
Mariflor AGUILAR \\ Universidad Nacional Autônoma de Méjico
}

\begin{abstract}
RESUMEN. El proceso de la comprensión incluye al menos tres momentos que en su conjunto arman sin duda una propuesta crítica: la exigencia de una mirada autorreflexiva de las propias expectativas de sentido; la exigencia hermenéutica de encontrar la diferencia con el interlocutor para que el diálogo pueda tener lugar y, por último, la modificación de mi perspectiva en tanto que he comprendido. Pensado así, con un concepto fuerte de "comprensión", el proceso hermenéutico promueve el círculo de correcciones y rectificaciones propuestas por la situación dialógica, y en esa misma medida, puede convertirse en un modelo de las interrelaciones o en una estrategia heurística que prevenga al intérprete de suprimir demasiado rápido las diferencias en favor de lo familiar.
\end{abstract}

Desde hace algunos años se han presentado varios intentos de pensar la hermenéutica como un instrumento filosófico útil para la crítica social. Se piensa, por ejemplo, en una hermenéutica crítica o en una genealogía hermenéutica que articula sobre todo a Gadamer con Foucault. Hay quienes también quieren ver en la hermenéutica una crítica radical a la razón del tipo de la de Nietzsche y la llamada posmodernidad, haciéndola partícipe de la misma fuerza crítica que se atribuye a estas teorías. Todo esto contrasta con uno de los cuestionamientos a la hermenéutica que más han circulado: la acusación de ser un pensamiento conservador y tradicionalista. No me voy a detener en el análisis de si la hermenéutica es o no un pensamiento de tipo posmoderno. Prefiero reflexionar acerca de qué es lo que hace posible que la hermenéutica se piense como útil para la crítica social a pesar del supuesto conservadurismo del que se le acusa, para lo cual me concentraré en dos tareas: primero, enfatizaré ciertas tesis que pueden ir en contra de la crítica de tradicionalismo y, segundo, intentaré mostrar que la estructura de la comprensión hermenéutica puede verse ella misma como una estructura relevante para el pensamiento crítico. 
Antes quisiera aclarar que no voy a entender por pensamiento "crítico" su sentido especulativo tal como pasó a la llustración y al idealismo alemán, sino más bien su sentido de emancipación práctica, es decir, de una reflexión que es útil para la clarificación de las luchas sociales. Es claro que una teoría útil para este tipo de reflexión no tiene que referirse ella misma a las luchas sociales. Una teoría puede considerarse útil para el pensamiento crítico si su aparato conceptual sirve, por ejemplo, para revelar ciertas estructuras de dominación. Esto es lo que creo que ocurre con la hermenéutica y lo que intentaré mostrar.

Pero comenzando por el principio, la acusación a Gadamer de tradicionalista se basa por lo general en un argumento que adopta formas diferentes. Mencionaré una de sus formas básicas y una derivada. La forma básica parte de la idea de que ser tradicionalista es considerar que un punto de vista tradicional es correcto no porque se haya juzgado reflexivamente como racionalmente aceptable sino simplemente porque es tradicional. Las consideraciones que acompañan la postura tradicionalista anterior suelen ser las siguientes: dado que somos históricamente finitos, dado que no tenemos un concepto de racionalidad que sea independiente de la tradición a la que pertenecemos y, por ende, no contamos con principios y normas universales a las cuales apelar, no debemos deshacernos de la autoridad de la tradición ${ }^{1}$. Puede ser claro que a esta postura que reconoce acríticamente la autoridad de la tradición puede llamársele "tradicionalista" y que desplazada a la política se trataría de una postura conservadora.

Una forma derivada de esta crítica se refiere también a la ausencia de puntos de vista universales desde los cuales criticar y, por lo tanto, a la imposibilidad de formular una crítica a las estructuras del poder que necesariamente conforman el plexo de la tradición. Esta forma derivada parte del supuesto de que lo que ocurre en la hermenéutica es del orden del «acontecer» de tipo heideggeriano, es decir, se trata de eventos que acaecen sin la participación consciente de los sujetos, se trata en suma de lo que ocurre a nuestras espaldas. Este planteamiento va como sigue: si cada interpretación está fundada en algún contexto particular y cada contexto puede ser permeado por estructuras de poder no reconocidas, se plantea entonces el problema de que no hay un punto arquimídeo desde el cual decidir lo que es el poder, un punto de vista que esté libre de toda estructura de

${ }^{1}$ Cfr. David C. HoY y Th. MCCARTHY, Critical Theory, Blackwell, Cambridge, 1994, p. 196. 
poder. ¿Cómo, entonces, puede evaluarse el poder en un sentido crítico y libe${\text { rador } ?^{2}}^{2}$

Puede observarse que en ambos casos la base de la crítica incluye dos elementos: la tradición y ciertos puntos de vista estables desde los cuales hacer la crítica. La tradición, en tanto se ha convertido en autoridad, sustituye los principios para toda evaluación.

Son muchos, en efecto, los lugares donde Gadamer se refiere a la imposibilidad de abandonar la tradición; y no sólo eso, sino que llega a afirmar que la tradición es el fundamento de la validez de las costumbres (VM, 348) ${ }^{3}$. La tradición como autoridad, los prejuicios como autoridad, son ciertamente tesis que forman el repertorio de frases comunes del Gadamer de divulgación. Lo que parece inferirse del inevitable rol que juega la tradición en la comprensión es que si no podemos escapar de ella tampoco tenemos derecho a criticarla. Y, sin embargo, no es tan obligado sacar esta conclusión. Mucho se ha escrito para refutar esta inferencia, empezando por el mismo Gadamer, quien se ha cansado de decir que la hermenéutica que él propone no va en contra de la posibilidad de criticar la tradición. Ha dicho también que no toda tradición es conservadora, sino que hay también tradiciones revolucionarias ${ }^{4}$, y habla también de las múltiples voces de la tradición. Por otra parte, es ya clásica la intervención de Paul Ricoeur en este debate en que sostiene que la crítica a la tradición se hace también desde otra tradición. En realidad, son varios los puntos de apoyo que se han encontrado dentro del discurso gadameriano para salvarlo de la acusación de tradicionalista, y uno de ellos es la redefinición de la noción misma de crítica.

Por más que, como decía, Gadamer se ha cansado de decir que su hermenéutica no va en contra de la posibilidad de criticar la tradición, tal parece que lo que entiende por crítica no convence a sus detractores. Y este me parece que es el punto central, pues se podría ver que detrás de la concepción de la tradición y de los prejuicios como autoridad se recorta una noción de crítica diferente. Se puede reinterpretar la tesis de la autoridad de la tradición como una tesis que invita a pensar la crítica como una actividad que no requiere de un pun-

\footnotetext{
${ }^{2}$ Cfr. H. H. KOGLer, The power of dialogue, MIT Press, 1996, p. 252.

${ }^{3}$ Así indicaremos la referencia a las páginas de Verdad y método, Sígueme, Salamanca, 1991.

${ }^{4}$ Verdad y método II, Sígueme, Salamanca, 1992, p. 260.
} 
to arquimídeo para llevarse a cabo sino más bien de una interacción con la pluralidad inscrita en el mismo proceso hermenéutico. Esta pluralidad está presente al menos en dos tesis: una es la de la circularidad de la comprensión; la otra es la de la pluralidad de voces de la tradición.

La idea de la circularidad de la comprensión en algún sentido impide pensar en el tradicionalismo en tanto que, según ella, aunque el contexto condiciona la comprensión, ésta a su vez condiciona el contexto ; es decir, puede verse el proceso hermenéutico de la comprensión como un proceso de resignificación incesante tanto del pasado como del presente que impide pensar en un concepto estático de tradición que no sea puesto al día constantemente en un movimiento de apertura y rectificación. Por otra parte, si tomamos seriamente en cuenta que la tradición es polifónica, puede pensarse que la condición de posibilidad de la crítica es precisamente la pluralidad: la adhesión a una instancia de la tradición supone en algún sentido el rechazo a otra. El fundamento de la crítica serían las voces diversas. La ausencia de un punto de partida por encima o por fuera de las múltiples voces de la tradición no haría entonces imposible la crítica. Por el contrario, estas voces se levantarán especialmente contra cualquier voz que pretenda ser la única autoridad porque ella sola representa la verdad de la tradición. En este sentido, la autoridad de la tradición debe verse como el reconocimiento de que "una sola persona o una sola situación discursiva no pueden iluminar completamente o justificar el mapa completo" ${ }^{6}$.

Hay que decir, sin embargo, que si bien es cierto que la tesis de la circularidad de la comprensión y de la pluralidad de voces de la tradición ayudan a pensar una noción de crítica compatible con la hermenéutica no representan, desde mi punto de vista, el aspecto crítico más importante de este proceso comprensivo. Hay un aspecto que no suele tomarse mucho en cuenta y que me parece que representa uno de los mayores logros de la hermenéutica, además de ser uno de los aspectos que, me parece, hacen que la estructura misma de la comprensión sea relevante para el pensamiento crítico. Me refiero a la reflexividad que acompaña a la exigencia hermenéutica de construir los horizontes históricos, tanto del intérprete como del interlocutor, como condición de posibilidad de la comprensión. Esta doble construcción de horizontes o de perspectivas, que en ver-

\footnotetext{
Cfr. David C. HoY, op. cit., p. 194.

${ }^{6}$ Ibíd., p. 197.
} 
dad constituye el origen del movimiento de la comprensión, suele tomarse en cuenta como un proceso que ocurre, como dije, como efecto del acontecer, es decir, como un proceso que remite a un "saber de fondo" compartido que, en cuanto tal, no puede ser traído a conciencia por los hablantes. Por este motivo se identifica la perspectiva de Gadamer con la de Heidegger en tanto que en ambos «la intersubjetividad necesaria para que una conversación tenga lugar sólo puede pensarse como una condición dada y no como resultado de un procedimiento a disposición de los hablantes que permitiera alcanzar un entendimiento desde un 'contexto cero', sin supuestos previos" ${ }^{7}$. Tiene razón Cristina Lafont en que en Gadamer, igual que en Heidegger, es impensable el "contexto cero", en la medida en que tradición y prejuicios son autoridad y son irrebasables. En lo que me parece que no tiene razón es en no ver, al menos en Gadamer, la dimensión de la reflexividad, que si bien no niega el contexto de la tradición sino que opera con él, es una dimensión tomada en cuenta como motor mismo de la comprensión. Trataré de mostrar esto.

El proceso de la comprensión incluye al menos tres momentos que me parecen relevantes: primero, la exigencia de una mirada autoreflexiva, de la conciencia de las propias expectativas de sentido acerca del objeto a comprender, conformadas por concepciones y creencias anticipadas que nos han sido transmitidas por enseñanzas y tradiciones diversas; segundo, la exigencia hermenéutica de "dialogar" con el otro, es decir, de tratar de comprenderlo desde ese horizonte previamente construido al mismo tiempo que se va construyendo su propio horizonte, es decir, al mismo tiempo que leo el discurso del otro como una respuesta a un plexo complejo de prácticas enraizadas en tradiciones; y tercero, los efectos que sobre mi propio horizonte tiene la contrastación de ambos horizontes. Me voy a referir brevemente a estos tres momentos.

En cuanto al primero, a la mirada autorreflexiva, me parece interesante que, al revés de lo que supondría el sentido común o un cierto sentido histórico, no se exija para comprender ponerse en el lugar del otro sino, antes al contrario, se exige más bien ponerse en el propio lugar, es decir, en la propia situación o perspectiva temporal desde la cual se comprende. Al ponernos en el lugar del otro en un movimiento unilateral cancelando nuestra perspectiva, se rompe la vin-

${ }^{7}$ Cristina Lafont, La razón como lenguaje, Visor, Madrid, 1993, p. 113. 
culatividad. Para comprender realmente se requiere la no autocancelación; así que una supuesta epojé del trasfondo previo de mi comprensión en lugar de hacerla posible la obstaculiza. Mediante la autocancelación del propio trasfondo no se logra realmente alcanzar la dimensión del otro. Siempre que se asume una postura unilateral, dice Gadamer, «se renuncia a la pretensión de hallar en la tradición una verdad comprensible que pueda ser válida para uno mismo" (VM, 374).

Lo que este proceso supone es una actividad por parte del intérprete que articula una responsabilidad y un esfuerzo. Una responsabilidad en la medida en que es consciente de que si ese trasfondo de creencias no es correcta o suficientemente construido la comprensión no podrá realizarse; y un esfuerzo, que se requiere siempre que se trata de comprender. La noción de "esfuerzo" es utilizada por Gadamer en distintos momentos clave como un recurso, pienso yo, para no hacer concesiones a la filosofía de la conciencia. Así, habla de esfuerzo hermenéutico en vez de la voluntad del intérprete o del propósito del intérprete de involucrarse de una u otra manera en la experiencia hermenéutica. En el caso de la construcción del propio horizonte histórico, el esfuerzo que se pone en juego es el de una autorreflexión que logre romper los lazos de conciencia y emotivos que nos atan a nuestras creencias comunes. Dice Gadamer: "Construir o ganar el horizonte propio requiere de un verdadero esfuerzo; requiere no quedarse atrapado por esperanzas y temores cercanos" (VM, 376).

Un aspecto que es interesante de este primer momento y de este primer esfuerzo de la comprensión es que su objetivo es alcanzar de la manera menos deformada posible el horizonte del otro. Cuanto mejor construya mi propio horizonte, mejor alcanzaré el otro horizonte, menos lo deformaré porque estaré en mejores condiciones para no proyectar injustificadamente sobre el otro mi propio trasfondo de creencias. Dice Gadamer: «Una conciencia formada hermenéuticamente tendrá que ser hasta cierto punto también conciencia histórica, y hacer conscientes los propios prejuicios que le guían en la comprensión con el fin de que la tradición se destaque a su vez como opinión distinta y acceda así a su derecho" (VM, 369). La diferencialidad de la tradición o de la voz del otro sólo puede destacarse sobre el trasfondo del horizonte de mis propios prejuicios. Paradójicamente, la condición de poner en suspenso mi propio horizonte como obstáculo para la comprensión es su construcción y la conciencia del mismo como condición para la comprensión. Esta actitud implica desde mi punto de vista la dimensión de la reflexividad y, con ella, una primera huella crítica que consiste 
en la disposición de poner entre paréntesis el propio horizonte, en la consideración de que él mismo es insuficiente para la comprensión del otro. Como vimos, es necesario pero también es insuficiente.

Por otra parte, el esfuerzo por construir el propio horizonte supone que el intérprete pueda darse cuenta de su situación, es decir, de su condición de ser situado, contextuado y condicionado por tradiciones diversas, lo cual abre desde mi punto de vista un espacio de desdoblamiento de la conciencia que puede traducirse también en una segunda marca crítica o quizá, mejor, de autocrítica, en tanto que a través del darse cuenta de la situación el intérprete puede también percatarse de cómo llegó a ser lo que es o a concebir lo que concibe sin su consentimiento ${ }^{8}$.

Este es un primer momento del proceso complejo de la comprensión. Para utilizar términos caros a Gadamer, este primer momento consiste en la construcción de lo que nos resulta familiar acerca de lo comprendido. El segundo momento consistirá en la construcción de lo extraño desde lo familiar. Si se logra el primer momento, si se consigue rebasar esperanzas y temores cercanos para construir responsablemente el propio horizonte de comprensión, se está entonces preparado para el segundo momento, que consiste, como dije, en intentar comprender o, como se dice en la jerga hermenéutica, en intentar escuchar la voz del otro. De lo que ahora se trata, dice Gadamer, es de "escuchar la tradición tal como ella puede hacerse oír en su sentido propio y diferente" (VM, 376).

Este sentido propio y diferente es lo que se trata de encontrar en el momento dialógico de la comprensión y lo que constituye la obsesión gadameriana: cómo percibir al otro como diferente desde nuestra propia comprensión; y en este lugar entra la apuesta de la reflexión hermenéutica: si hemos construido adecuadamente nuestro horizonte histórico, tarde o temprano se manifestará la diferencia del otro. En algún punto, en algún momento, el texto no hará sentido, nos sorprenderá o chocará con nuestras expectativas. Ese es el punto en el que se desencadena la comprensión del otro y el punto en el que, según creo, aparece una tercera marca crítica que consiste en percibir al otro desde su propio horizonte, escucharlo y comprenderlo a partir de la construcción que vamos hacien-

${ }^{8}$ Cfr. H. H. KOGLER, op. cit., p. 268. 
do de su propio horizonte desde lo que él dice y desde lo que nosotros no somos. Como dije antes, este segundo momento representa la dimensión de lo extraño, con lo cual se abarca lo que Gadamer llama "el verdadero tópos de la hermenéutica», que es la posición entre extrañeza y familiaridad. $\mathrm{Al}$ respecto dice también Gadamer: «Existe una verdadera polaridad de familiaridad y extrañeza, y en ella se basa la tarea de la hermenéutica, pero no en el sentido psicológico de Schleiermacher, como el ámbito que oculta el misterio de la individualidad, sino en un sentido verdaderamente hermenéutico, esto es, con la atención puesta en algo dicho: el lenguaje en el que nos habla la tradición» (VM, 365).

Por otra parte, en cuanto al tercer momento, el de los efectos del diálogo comprensivo sobre mi propio paquete horizóntico, encierra uno de los mayores momentos de la crítica. No sólo se parte del supuesto de que el otro puede ser diferente, sino también de que el otro puede modificar mi horizonte, de que mis creencias heredadas por tradición no son definitivas y pueden ponerse en cuestión. Una consecuencia posible de haber logrado comprender al otro en su diferencia es que el intérprete puede leerse a sí mismo desde el punto de vista del otro'. La perspectiva desde el punto de vista del otro arroja una luz específica sobre nosotros mismos que no hubiéramos podido generar por nosotros mis$\operatorname{mos}^{10}$. De aquí la importancia de este momento para la reflexividad. La situación dialógica es relevante no solamente para la percepción del otro en sus diferencias, sino también por la producción de una reflexividad más compleja que hace posible un distanciamiento de la propia situación.

A partir de la revelación del otro como diferente, el esfuerzo no tiene por qué dirigirse a equiparar al otro con mi perspectiva, es decir, no tiene que dirigirse a hacer de lo extraño algo familiar sino, por el contrario, el choque del texto o de lo otro con mis expectativas puede obligar a que incluso lo familiar que creo ver se transforme en algo extraño ${ }^{11}$; es decir, frente a lo diferente la posibilidad no es borrar las diferencias para subordinarlas a mi perspectiva; se puede simple-

9 Cfr. Thomas M. AleXANDER, «Eros and understanding: Gadamer's aesthetic ontology of the communitym, en Lewis Edwin Hahn (ed.), The Philosophy of Hans-Georg Gadamer, The Library of Living Philosophers, Southern Illinois University, 1997, p. 328.

${ }^{10}$ Cfr. H. H. KÖler, op. cit., p. 252.

${ }^{11}$ Cfr. sobre este punto D. C. HoY, op. cit., p. 192 e ibíd., "Post-cartesian interpretation: HansGeorg Gadamer and Donald Davidson", en Lewis Edwin Hahn (ed.), op. cit. 
mente admitir la diferencia de ambos puntos de vista y hasta la posibilidad de que la perspectiva del otro fuerce a criticar mis propias perspectivas ${ }^{12}$. Como señala David Couzens Hoy, estudioso de Gadamer, la comprensión hermenéutica puede requerir una confrontación con lo extraño precisamente para alejarnos de la complacencia con nuestro propio punto de vista ${ }^{13}$.

En suma, el montaje hermenéutico aporta a la teoría crítica la posibilidad de pensar la comprensión de los significados como enraizados en la historia y en tradiciones y a partir de ahí la posibilidad de revisar las propias precomprensiones. Esta manera de ver la hermenéutica enfatiza la lectura gadameriana que da lugar a la reflexividad en lugar de aquélla que presenta el proceso de la comprensión como un fenómeno del acontecer, de lo que ocurre a nuestras espaldas. $Y$ este me parece que es el mérito de Gadamer, mostrar que si no podemos prescindir del acontecer de la tradición que produce inevitablemente sus efectos sobre nuestra comprensión, es entonces mejor aliarnos reflexivamente con él para de esta manera incidir parcialmente en sus efectos.

Pensada la hermenéutica como crítica se reabre el espacio para la reflexión en la cual los sujetos pueden reconceptualizar sus identidades observando sus propios trasfondos dados de antemano como construcciones sociales ${ }^{14}$. En la medida en que este montaje parte del supuesto de que el otro puede ser diferente y busca construir el armazón requerido para no perder estas diferencias, en esa medida la hermenéutica crítica se convierte en un modelo de las interrelaciones o bien en una estrategia heurística que previene al intérprete de suprimir demasiado rápido las diferencias a favor de lo familiar ${ }^{15}$.

${ }^{12}$ Cfr. sobre este punto D. C. HoY, en D.C. Hoy, Th. McCarthy, op. cit., p. 202.

${ }^{13}$ David C. HoY, “Post-cartesian...", loc. cit., p. 124.

${ }^{14}$ H. H. KOGLER, op. cit., p. 235.

${ }^{15}$ D. C. HoY, en D. C. Hoy y Th. McCarthy, op. cit., p. 174. 